\title{
ON A UBIQUITOUS CARDINAL ${ }^{1}$
}

\author{
STEPHEN H. HECHLER
}

\begin{abstract}
We consider five combinatorial or topological structures, each with a certain associated minimal cardinal, and we show that these cardinals are always equal even though it is independent of the axioms of set theory as to just what the value of this common cardinal is. The five structures are the set of functions from $N$ (the set of natural numbers) into $N$ under two partial orderings, the rational numbers with respect to closed embeddings into powers of $N$, a certain subset of $\beta N-N$ with respect to clopen decompositions, the irrationals with respect to compact decompositions, and a subclass of the Borel sets with respect to closed decompositions. The proofs presented do not require a knowledge of forcing techniques.
\end{abstract}

In this paper we shall consider five different topological or combinatorial structures and certain minimal cardinals associated with each of these. We shall show that although it is consistent with the axioms of ZermeloFraenkel set theory that any one of these cardinals takes on any value less than or equal to $\mathbf{c}$ (the cardinality of the continuum) subject only to the restriction that it have uncountable cofinality, the five cardinals in question are, nevertheless, always equal. While we shall refer to independence proofs elsewhere, we shall not deal with any here, and, in particular, we shall not use forcing techniques.

The five structures and their associated cardinals are:

1. Let $N$ be the set of natural numbers, and let ${ }^{N} N$ be the set of functions from $N$ into $N$. We define partial orderings $<_{1}$ and $<_{2}$ on ${ }^{N} N$ by setting:

$$
\begin{aligned}
& f<_{1} g \leftrightarrow \forall n[f(n)<g(n)], \\
& f<_{2} g \leftrightarrow \exists k \forall n>k[f(n)<g(n)],
\end{aligned}
$$

and for $i=1,2$ we define a set $\mathcal{S} \subseteq{ }^{N} N$ to be an i-scale iff $f \in{ }^{N} N \rightarrow$ $\exists g \in \mathcal{S}\left[f<_{1} g\right]$. Finally, we define $\mathbf{K}_{1}^{i}$ to be the least cardinal $\kappa$ such that there exists an $i$-scale of cardinality $\kappa$.

Lemma. The cardinals $\mathbf{K}_{1}^{1}$ and $\mathbf{K}_{1}^{2}$ are always equal.

Proof. Clearly, any 1-scale is a 2 -scale, and if $\mathfrak{d}$ is any 2 -scale, then

Received by the editors July 30, 1974.

AMS (MOS) subject classifications (1970). Primary 02K25, 04A30, 54D99, 54H99, 54A25; Secondary 02K30, 04A15, 54H05.

${ }^{1}$ The preparation of this paper was partially supported by a grant from the City University of New York Faculty Research Program. 


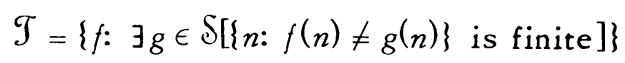

is a 1-scale of cardinality equal to that of $\mathcal{S}$.

We shall refer to this cardinal as $K_{1}$.

2. Let $\mathbf{N}$ be the space obtained by putting the discrete topology on $N$, for any cardinal $\kappa$, let $\mathbf{N}^{\kappa}$ be the topological product of $\kappa$ copies of $\mathbf{N}$, and let $\mathbf{Q}$ be the space consisting of the rational numbers with the inherited topology. Then it is known [5], [10] that $\mathbf{Q}$ can be embedded as a closed subset of $\mathbf{N}^{\kappa}$ for large enough $\kappa$. Let $\mathbf{K}_{2}$ be the smallest such cardinal.

3. Let $\beta \mathbf{N}$ be the Stone-Čech compactification of $\mathbf{N}$, and let $\mathcal{B}$ be the family of clopen subsets of $\beta \mathbf{N}-\mathbf{N}$. It is well known that $\mathcal{B}$ is a base for the topology of $\beta N-N$, so, following Negrepontis [11], it is reasonable to define the type of an open set $U \subseteq \beta \mathbf{N}-\mathbf{N}$ to be the smallest cardinal $\kappa$ for which there exists a family $\mathcal{U} \subseteq \mathcal{B}$ of cardinality $\kappa$ such that $\cup \mathcal{U}=U$. It should be noted that since the members of $B$ are compact, any clopen cover of an open set of infinite type admits a subcover of cardinality equal to the type of the covered set. Now let $S$ be an open set of the simplest infinite type, namely of type $\boldsymbol{N}_{0}$. It is easily seen that not only are any two sets of this type homeomorphic, but such a homeomorphism can always be found which is induced by a permutation of $N$ and which, therefore, can be extended to all of $\beta \mathbf{N}-\mathbf{N} .^{2}$ So $S$ is, in a strong sense, unique, and thus so is its exterior (the interior of its complement). Let $K_{3}$ be the type of the exterior of $S$.

4. Let I be the space consisting of the irrational numbers with the inherited topology, and let $\mathcal{C}$ be the family of compact subsets of I. Note that a set belongs to $\mathcal{C}$ iff it is closed, bounded, and remains closed when considered as a subset of the real line. Define $\mathbf{K}_{4}$ to be the smallest cardinal $\kappa$ for which there exists a family $\mathcal{F} \subseteq \mathcal{C}$ of cardinality $\kappa$ which covers $\mathbf{I}$.

5. Let $\mathbf{R}$ be the real line with the usual topology, and let $\mathbf{F}$ and $\mathbf{G}$ be the families of closed and open subsets of $\mathbf{R}$ respectively. Then define a set to be an elementary Borel set iff it can be obtained from $\mathbf{F}$ or $\mathbf{G}$ by a finite sequence of the operations, countable union and countable intersection. Thus a set is elementary Borel iff it is in one of the classes $\mathbf{F}, \mathbf{G}, \mathbf{F}_{\sigma}, \mathbf{G}_{\delta}$, $\mathbf{F}_{\sigma \delta}, \mathbf{G}_{\delta \sigma}, \mathbf{F}_{\sigma \delta \sigma}, \cdots$, where the indices are all finite. Define $\mathbf{K}_{5}$ to be the smallest cardinal $\kappa$ such that every elementary Borel set can be ex-

\footnotetext{
${ }^{2}$ In fact, M. E. Rudin [12] has shown that given the continuum hypothesis any homeomorphism between two such sets can be extended to all of $\beta N-N$. For details and generalizations see also [3].
} 
pressed as a union of $\kappa$ closed sets. We note that since every closed subset of $\mathbf{R}$ is a countable union of compact subsets, we may replace "closed" by "compact" in this definition.

Although these cardinals appear, for the most part, to be quite unrelated, we show that this is far from the case. In fact, our main theorem, which we prove next, tells us that they are all equal.

Theorem 1. The cardinals $\mathbf{K}_{1}, \mathbf{K}_{2}, \mathbf{K}_{3}, \mathbf{K}_{4}$, and $\mathbf{K}_{5}$ are all equal.

Proof. The proof that $K_{1}=K_{3}$ can be found in [8], and the proof that $K_{2}=K_{4}$ is given in [5]. Here we shall prove $K_{1}=K_{4}$ and $K_{4} \leq K_{5} \leq K_{1}$.

We begin by proving $K_{1}=K_{4}$ using the well-known fact that $I$ is homeomorphic to $\mathbf{N}^{N}$. This allows us to think of the points of $I$ as functions from $N$ into $N$. For each $n \in N$ let $\pi_{n}$ be the projection function from $N^{N}$ onto the $n$th term of the product. Now suppose that $C$ is any compact subset of $\mathbf{N}^{N}$. Then for each $n \in N$ the image $\pi_{n}[C]$ of $C$ under $\pi_{n}$ must be compact and, therefore, finite. Thus for each set $C \in \mathcal{C}$ we may define a function $f_{C} \in{ }^{N} N$ by setting $f_{C}(n)=\max \left(\pi_{n}[C]\right)$. Similarly, for each function $f \in{ }^{N} N$ we may define a compact set $C_{f} \in \mathcal{C}$ by $C_{f}=\left\{g \in N^{N}: g \leq_{1} f\right\}$. But now it is easily seen that if $U \subseteq \mathcal{C}$ is a cover of $\mathbf{N}^{N}$, then $\left\{f_{C}: C \in \mathcal{U}\right\}$ is a 1-scale, while if $\mathcal{S}$ is any 1-scale, then $\left\{C_{f}: f \in \mathcal{S}\right\}$ is a cover of $\mathbf{N}^{N}$. We are greatly indebted to $S$. Mrówka for suggesting that we consider this characterization of $\mathbf{I}$ and its combinatorial consequences.

Since $\mathbf{I}$ is a $\mathbf{G}_{\delta}$ set, and since any subset of $\mathbf{I}$ which is closed in $\mathbf{R}$ is a countable union of compact subsets of $\mathbf{I}$, it is clear that $\mathbf{K}_{4} \leq \mathbf{K}_{5}$. To prove $K_{5} \leq K_{1}$, we require a technique used by Chambers [1] to deal with certain uncountable arrays. We, however, shall apply it to countable arrays, but because ours will be an induction proof, we shall, in effect, use it infinitely many times.

To carry out our proof, we note that since an open set is also an $\mathbf{F}_{\sigma}$ set, we need only consider the classes $\mathbf{F}, \mathbf{F}_{\sigma}, \mathbf{F}_{\sigma \delta}, \mathbf{F}_{\sigma \delta \sigma}$, etc., and it is also clear that we need not worry about classes $\mathbf{F}_{a}$ where the last term in $\alpha$ is $\sigma$. We shall, therefore, carry out our induction of the number $n$ of occurrences of $\delta$ in $a$. The theorem is true for $n=0$, so suppose it is true for $n \leq k$, and consider a set $A$ in the class $\mathbf{F}_{a \sigma \delta}$ where $\alpha$ is either empty or a string of $2 k$ symbols the last of which is $\delta$. Then we may write

$$
A=\bigcap_{i \in N} \bigcup_{j \in N} F_{j}^{i}
$$

where each $F_{j}^{i}$ is an $F_{a}$ set. Furthermore, since Borel classes are all closed under finite unions, we may assume that for any $i, j, k \in N$ we have $F_{j}^{i} \subseteq$ $F_{j+k}^{i}$. Thus, if for each point $p \in A$ we define a function $f_{p}$ by setting $f_{p}(i)$ $=\min \left\{j: p \in F_{j}^{i}\right\}$, we have 


$$
f_{p} \leq_{1} g \rightarrow p \in \bigcap_{i \in N} F_{g(i)}^{i}
$$

Next, let $\mathcal{S}$ be any 1 -scale of cardinality $K_{1}$. Then from the above, we see that

$$
A=\bigcup_{g \in \mathcal{S}} \bigcap_{i \in N} F_{g(i)}^{i}
$$

and for each function $g \in \mathcal{S}$ we define $F_{g}=\bigcap_{i \in N} F_{g(i)}^{i}$. Since each $F_{g(i)}^{i}$ is an $\mathbf{F}_{\alpha}$ set, and the last symbol in $\alpha$ (if $\alpha$ is not empty) is a $\delta$, each $F_{g}$ is also an $\mathbf{F}_{a}$ set. But now, by the induction hypothesis, each $F_{g}$ is a union of $\mathrm{K}_{1}$ closed sets, and, therefore, so is $A$.

Since we have now shown them to be equal, we shall use $\mathbf{K}$ to denote all of the $K_{i}$. We next consider the possible values $\mathbf{K}$ can take on. Obviously, $\mathbf{K}$ cannot be greater than $\mathbf{c}$, and by looking at 2-scales, we show that it must have uncountable cofinality (i.e. that it cannot be expressed as a countable union of smaller cardinals).

Theorem 2. If any 2-scale $\mathcal{S}$ is decomposed into a countable collection $\left\{\mathcal{S}_{n} \subseteq \mathcal{S}: n \in N\right\}$ of not necessarily disjoint pieces, then at least one of the $\mathcal{S}_{n}$ must itself be a 2-scale.

Proof. Suppose otherwise. Then for each $n \in N$ there is a function $f_{n} \in{ }^{N} N$ such that for no $g \in \mathcal{S}_{n}$ is it the case that $f_{n}<_{2} g$. Now define a function $f$ by setting $f(n)=\sum_{j=1}^{n} f_{j}(n)$. Clearly, there cannot exist a function $g \in \mathcal{S}$ such that $f<_{2} g$.

Corollary. The cofinality of $\mathbf{K}$ must be greater than $\boldsymbol{N}_{0}$.

However, this is just about all we can say definitely about K. Elsewhere $[8]$, we have proven

Theorem 3. It is consistent with the axioms of Zermelo-Fraenkel set theory that $\mathbf{K}$ be any cardinal of uncountable cofinality which is less than or equal to c.

In particular, in Solovay's [13] models in which random reals are used it is known that $\mathbf{K}$ is equal to $\boldsymbol{N}_{1}$, while in Cohen's [2] models containing generic reals and in models where Martin's Axiom [9] holds, $\mathbf{K}$ is equal to c. For more on the possible structure of 2-scales see [7], and for discussions of various related cardinals see [6] and [14].

We conclude with two open problems.

Problem 1. Can our results on elementary Borel sets be extended to all Borel sets? S. Shelah in a private communication has shown that they can be if $\mathbf{K}$ is less than $\boldsymbol{N}_{\omega}$, but nothing is known for larger $\mathbf{K}$.

Problem 2. Let $\mathbf{K}^{*}$ be the number of nowhere dense sets needed to 
cover $\mathbf{R}$. Then since each member of $\mathcal{C}$ is nowhere dense in $\mathbf{R}$, we have $\mathbf{K}^{*} \leq \mathbf{K}+\boldsymbol{K}_{0}=\mathbf{K}$. Does $\mathbf{K}^{*}=\mathbf{K}$ ? If not, can $\mathbf{K}^{*}$ have cofinality $\boldsymbol{K}_{0}$ ? (For independence results concerning $K^{*}$ see [4].)

\section{REFERENCES}

1. M. Chambers, Sur le problème du continu et les échelles bien ordonnées pour les suites transfinies, Doctoral Dissertation, Université de Montréal.

2. P. J. Cohen, Set theory and the continuum hypothesis, Benjamin, New York, 1966. MR 38 \#999.

3, W. W. Comfort and S. Negrepontis, The theory of ultrafilters, SpringerVerlag, Berlin and New York, 1974.

4. S. H. Hechler, Independence results concerning the number of nowhere dense sets needed to cover the real line, Acta Math. Acad. Sci. Hungar. 24 (1973), 27-32. MR 47 \#1611. 395.

5. - Exponents of some N-compact spaces, Israel J. Math. 15 (1974), 384-

6. - A dozen small uncountable cardinals, TOPO 72-General Topology and its Applications, Lecture Notes in Math., vol. 378, Springer-Verlag, Berlin and New York, 1974, pp. 207-218.

7. - On the existence of certain cofinal subsets of ${ }^{\omega} \omega$, Proc. Sympos. Pure Math., vol. 13, part 2, Amer. Math. Soc., Providence, R.I., 1974, pp. 155-173.

8. - On the structure of a certain open subset of $\beta N-N$ (to appear).

9. D. A. Martin and R. M. Solovay, Internal Cohen extensions, Ann. Math. Logic 2 (1970), 143-178. MR 42 \#5787.

10. S. Mrówka, Further results on E-compact spaces. I, Acta Math. 120 (1968), 161-185. MR 37 \#2165.

11. S. Negrepontis, Extension of continuous functions in $\beta D$, Nederl. Akad. Wetensch. Proc. Ser. A $71=30$ (1968), 393-400. MR 39\#2128.

12. M. E. Rudin, Types of ultrafilters, Topology Seminar (Wisconsin, 1965), Ann. of Math. Studies, no. 60, Princeton Univ. Press, Princeton, N. J., 1966, pp. 147-151. MR 35\#7284.

13. R. M. Solovay, A model of set theory in which every set of reals is Lebesgue measurable, Ann. of Math. (2) 92 (1970), 1-56. MR 42 \#64.

14. F. Tall, An alternative to the continuum hypothesis and its uses in general topology (preprint).

DEPARTMENT OF MATHEMATICS, QUEENS COLLEGE, CITY UNIVERSITY OF NEW YORK, FLUSHING, NEW YORK 11367 